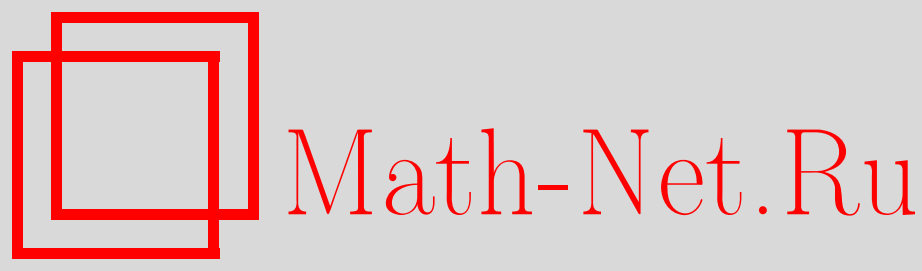

H. Möller, An Efficient Reliable Algorithm for the Approximation of All Polynomial Roots Based on the Method of D. Bernoulli, Совр. пробл. матем., 2012, выпуск $16,52-65$

DOI: https://doi.org/10.4213/spm34

Использование Общероссийского математического портала Math-Net.Ru подразумевает, что вы прочитали и согласны с пользовательским соглашением http://www.mathnet.ru/rus/agreement

Параметры загрузки:

IP : 35.173 .219 .149

26 апреля 2023 г., 12:41:45

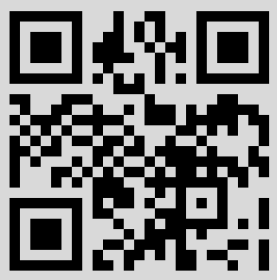




\title{
An Efficient Reliable Algorithm for the Approximation of All Polynomial Roots Based on the Method of D. Bernoulli
}

\author{
H. Möller \\ Mathematisches Institut, Westfälische Wilhelms-Universität Münster, Germany
}

In memory of A. A. Karatsuba, my mentor of number theory in 1970

\section{Introduction}

The design of the complete algorithm is based on eight theorems. Since most of them share the same conditions and abbreviations, we first state these specifications. Then we summarize the contents of this paper and put them in order with the efficient reliable polynomial rootfinding methods already known.

Specifications. Let $\mathcal{J}_{m}:=\{1, \ldots, m\}$ for $m \in \mathbb{N}_{1}:=\{1,2, \ldots\}$ and let $z_{1}, \ldots, z_{k}, k \in \mathbb{N}_{1} \backslash\{1\}$, be complex numbers with $z_{g} \neq z_{h}$ for $g, h \in \mathcal{J}_{k}, g \neq h$, and $0<\left|z_{1}\right| \leqslant \cdots \leqslant\left|z_{k}\right|$. A polynomial $p(z)$ is given by $p(z)=\sum_{i=0}^{m} a_{i} z^{i}$ with $a_{i} \in \mathbb{C}, i \in \mathcal{J}_{m}, a_{m} \neq 0$ and $a_{0}=-1$. It denotes either the original polynomial, a shift of it or a polynomial obtained by deflation. In the product representation $p(z)=a_{m} \prod_{j=1}^{k}\left(z-z_{j}\right)^{m_{j}}$ with $m_{j} \in \mathbb{N}_{1}$ the roots $z_{1}, \ldots, z_{k}$ are complex numbers with properties specified above. If not stated otherwise, the index $j$ ranges in $\mathcal{J}_{k}$, and the index $n$ is in $\mathbb{N}_{0}:=$ $\mathbb{N}_{1} \cup\{0\}$. For example, $\left(q_{n}\right)_{n}$ denotes the sequence $\left(q_{n}\right)_{n \in \mathbb{N}_{0}}$.

Throughout this paper the following abbreviations are used:

$$
\begin{gathered}
s_{n}:=\sum_{j=1}^{k} m_{j} z_{j}^{-n}, \quad k(n):=\min \left\{k \in \mathbb{N}_{1} \mid k>n \text { and } s_{k} \neq 0\right\}, \quad q_{n}:=\frac{s_{n}}{s_{k(n)}}, \\
\mathcal{Z}:=\left\{z_{1}, \ldots, z_{k}\right\}, \quad \mathcal{Z}_{1}:=\left\{z \in \mathbb{C} \mid p^{\prime}(z)=0\right\}, \\
\mu_{j}:=\min \left\{d \in \mathbb{R} \mid \text { there is a } g \in \mathcal{J}_{k} \backslash\{j\} \text { with } d=\left|z_{g}-z_{j}\right|\right\}, \quad \mu:=\min \left\{\mu_{1}, \ldots, \mu_{k}\right\}, \\
h_{t}(u):=u-t \frac{p(u)}{p^{\prime}(u)}, \quad \text { for } \quad t \in \mathcal{J}_{m} \cup\left\{\frac{m}{2}\right\}, \quad u \in \mathbb{C} \backslash \mathcal{Z}_{1} .
\end{gathered}
$$

In Section 2 we start with Newton's formulas connecting the sums of powers $s_{n}$ with the coefficients of $p(z)$. Since the sums of powers are fundamental for the mathematical derivation given here, the method is called Sums of Powers Algorithm (SPA). The essential theoretical results were published in [1]. But many of the facts which let the corresponding rootfinding program fulfill the criteria of [2] have been developed and tested by D. Gunsthövel who planned to write a dissertation in this field. For example, in Theorem 1 he has found the explicit a priori lower bound for the number of steps needed to get the desired accuracy depending on a fixed upper bound of $\left|z_{1} / z_{2}\right|$.

The basic recursion formula for $q_{n}$ is stated in Theorem 2. This is one of six results which in similar form are also contained in [1].

In Section 3 the error bounds and two stopping criteria are developed. They are connected with "Laguerre disks" which, by definition, are the closed disks having any $u \in \mathbb{C} \backslash\left(\mathcal{Z} \cup \mathcal{Z}_{1}\right)$ and $h_{m}(u)$ 
as endpoints of a diameter. It is known from function theory that every Laguerre disk contains at least one root of $p(z)$. Moreover, the radius is bounded from above and below by constant multiples of the distance between $\{u\}$ and $\mathcal{Z}$ if the distance is sufficiently small.

In Section 4 the "second phase" of the SPA is established which solves the convergence problem of the Bernoulli method if $\left|z_{1} / z_{2}\right|$ is close or equal to 1 . At first, the minimal absolute value of the roots is approximated by a number $r$ only using the quotients $q_{n}$ calculated in the "first phase". Afterwards, local minima of

$$
\left(t \rightarrow|p(r \cos t+i r \sin t)|^{2}, t \in[0,2 \pi[)\right.
$$

are determined with the help of the Fourier expansion of this function. Then, after shifting to the corresponding points in $\mathbb{C}$, the SPA gives quotient sequences which normally converge sufficiently fast to roots with absolute values close to $r$.

In Section 5 "chained minimum circles and modified Turán circles" are used to handle the "ultimate case". In this way, the SPA becomes absolutely reliable with good worst case complexity. Moreover, the interplay of quotient sequences and Laguerre disks enables us in Section 6 to separate all roots in disjoint disks and to overcome the deflation error. The last part is devoted to Newton-Raphson iteration. The same condition which has been used for the first stopping criterion in Section 3 now ensures at least quadratic convergence of the sequence $\left(u_{n}\right)_{n}$ generated by $u_{n+1}:=h_{1}\left(u_{n}\right), n \in \mathbb{N}_{0}$, if the single root contained in the Laguerre disk of $u_{0}$ has multiplicity 1 .

Several survey articles and bibliographies on polynomial rootfinding have been published during the last thirty years (e.g. [3]). Therefore, only some supplementary remarks shall help to compare the SPA with efficient reliable methods already known. The properties of the SPA are similar to those of the Jenkins-Traub algorithm [4] which indeed starts with a reformulation of Bernoulli iteration. There are two major differences, namely, the SPA doesn't directly lead to quadratic convergence, but it makes up for this by not depending on any unsure decisions. In fact, it can be used to determine the critical number $s$ in Stage Two of the Jenkins-Traub algorithm. As well, it is able to produce satisfactory starting values for the Dochev-Durand-Kerner-Weierstrass algorithm [5] which simultaneously determines approximations for all roots.

Since the SPA immediately works with numbers which have the same order as the roots, its efficiency is quite good compared with other methods which use disks or circles, namely, the Cohn-Lehmer-Schur algorithm [6] which encloses the roots by two-dimensional bisection and the Henrici-Schönhage-Weyl algorithm [7] which factors polynomials with the help of contour integrals.

\section{Properties of sums of powers}

If $p(z)$ is a polynomial as specified above, then the sums of powers $s_{n}$ for $n \in \mathbb{N}_{1}$ can be determined recursively with Newton's formulas:

$$
s_{n}= \begin{cases}\sum_{j=1}^{n-1} a_{j} s_{n-j}+n a_{n} & \text { when } n \leqslant m, \\ \sum_{j=1}^{m} a_{j} s_{n-j} & \text { when } n>m .\end{cases}
$$

THEOREM 1. Let $z_{1}, \ldots, z_{k}$ be complex numbers as specified above with $\left|z_{1}\right|<\left|z_{2}\right|$ and $\left|z_{1}\right| \leqslant b$, where $b$ may be chosen suitably. If $\varepsilon$ and $\varkappa$ are real numbers with $0<\varepsilon \leqslant 2 b$ and $\left|z_{1} / z_{2}\right| \leqslant \varkappa<1$, then $\left|q_{n}-z_{1}\right|<\varepsilon$ holds for every $n \in \mathbb{N}_{0}$ with

$$
n \geqslant\left[\left(\log \varkappa^{-1}\right)^{-1} \log \left(4 m b \varepsilon^{-1}\right)\right]+1 .
$$


Proof. If $k=1$, then we have $s_{n}=m z_{1}^{-n}$ and therefore $q_{n}=z_{1}$ for every $n \in \mathbb{N}_{0}$. In the case $k>1$ we write $s_{n}=z_{1}^{-n}\left(m_{1}+m_{2} \zeta_{2}^{n}+\cdots+m_{k} \zeta_{k}^{n}\right)$ with $\zeta_{j}:=z_{1} / z_{j}$ for $j=2, \ldots, k$. Since $\left|m_{2} \zeta_{2}^{n}+\cdots+m_{k} \zeta_{k}^{n}\right| \leqslant m \varkappa^{n}$, it follows that

$$
\left|m_{1}+m_{2} \zeta_{2}^{n}+\cdots+m_{k} \zeta_{k}^{n}\right| \geqslant \frac{1}{2} \quad \text { for all } \quad n \geqslant \frac{\log (2 m)}{-\log \varkappa}=: n_{0} .
$$

In particular, we get $s_{n} \neq 0$ and therefore $k(n)=n+1$ for $n \geqslant n_{0}$. For these $n$, we have

$$
q_{n}-z_{1}=z_{1}\left(\sum_{j=2}^{k} m_{j} \zeta_{j}^{n}\left(1-\zeta_{j}\right)\right)\left(m_{1}+\sum_{j=2}^{k} m_{j} \zeta_{j}^{n+1}\right)^{-1}
$$

from which $\left|q_{n}-z_{1}\right| \leqslant\left|z_{1}\right| 4 m \varkappa^{n}$ follows. Hence for every $n \in \mathbb{N}_{0}$ with

$$
n>\frac{\log \left(4 m b \varepsilon^{-1}\right)}{\log \varkappa^{-1}} \geqslant n_{0}
$$

we get $\left|q_{n}-z_{1}\right|<\varepsilon$.

Based on extensive tests, $(\log 1 / 0.71 \ldots)^{-1}=3.0$ has been chosen as the first factor of $(2)$ for the implementation of the SPA. This means that the number of steps on the right-hand side of (2) will give the desired accuracy if $\left|z_{2}\right| \geqslant 1.4\left|z_{1}\right|$. As derived in Section 4, a failure is not disadvantageous because the quotients $\left|z_{1} / z_{2}\right|$ can be decrease with the help of suitable substitutions. Then this "stepfactor" will be chosen as 2.0 which is equivalent with $\left|z_{2}\right| \geqslant 1.65\left|z_{1}\right|$. The tests have led to the insight that a relatively small stepfactor is sufficient for the efficiency and reliability of the SPA independently of the type of the polynomial. This can also be verified with the program [9].

Since $\left(s_{n}\right)_{n}$ or $\left(s_{k(n)}^{-1}\right)_{n}$ may be unbounded, even if $\left(q_{n}\right)_{n}$ is bounded, a new recursion formula for $q_{n}$ is decisive for the efficiency of the SPA. Each sequence $\left(q_{n}\right)_{n}$ is called Bernoulli sequence. For instance, in the second case of (1) with $s_{k} \neq 0$ for every $k \in \mathbb{N}_{1}$ it follows that

$$
\begin{aligned}
q_{n}^{-1} & =s_{n+1} s_{n}^{-1}=a_{1}+a_{2} s_{n-1} s_{n}^{-1}+\cdots+a_{m} s_{n+1-m} s_{n}^{-1} \\
& =a_{1}+a_{2} q_{n-1}+\cdots+a_{m} q_{n+1-m} \cdots q_{n-2} q_{n-1}=a_{1}+\left(a_{2}+(\ldots) q_{n-2}\right) q_{n-1} .
\end{aligned}
$$

Theorem 2. Let $p(z)$ be a polynomial as specified above. Then $k(0)=\min \left\{k \in \mathcal{J}_{m} \mid a_{k} \neq 0\right\}$ and $q_{0}=m\left(k(0) a_{k(0)}\right)^{-1}$. Assume $k(s)$ and $q_{s}$ to be known for $s=\max \{0, n-m\}, \ldots, n-1$. Then $k(n)$ and $q_{n}$ can be calculated as follows.

For $s=\max \{0, n-m\}, \ldots, n-1$ and for $t \in \mathcal{J}_{m}$, introduce

$$
q_{s}^{(s+t)}:=\left\{\begin{array}{ll}
q_{s} & \text { when } s>0, q_{s} \neq 0, \\
1 & \text { when } s>0, q_{s}=0, \\
t\left(k(0) a_{k(0)}\right)^{-1} & \text { when } s=0,
\end{array} \quad a_{t}^{(s+t)}:= \begin{cases}a_{t} & \text { when } q_{s} \neq 0, \\
0 & \text { when } q_{s}=0 .\end{cases}\right.
$$

In the case $k(n-1)=n$, define

$$
p_{n, k}:=a_{k-n}+\sum_{t=k-n+1}^{\min \{k, m\}}\left(a_{t}^{(k)} \prod_{s=k-t}^{n-1} q_{s}^{(k)}\right)
$$

for $k=n+1, \ldots, n+m$. Then $k(n)=n+\min \left\{j \in \mathcal{J}_{m} \mid p_{n, n+j} \neq 0\right\}$ and $q_{n}=p_{n, k(n)}^{-1}$ hold. For $k(n-1)>n$, it follows that $k(n)=k(n-1)$ and $q_{n}=0$. 
Proof. Let $r:=\min \left\{k \in \mathcal{J}_{m} \mid a_{k} \neq 0\right\}$. Since $1 \leqslant r \leqslant m$, we have

$$
s_{r}=\sum_{j=1}^{r-1} a_{j} s_{r-j}+r a_{r}=r a_{r} \neq 0
$$

and $s_{n}=0$ for $1 \leqslant n<r$. Thus it follows that $k(0)=r$ and $q_{0}=m s_{r}^{-1}=m\left(r a_{r}\right)^{-1}$.

The condition $k(n-1)=n$ is equivalent with $s_{n} \neq 0$. Then, for $k=n+1$, we get

$$
s_{k} s_{n}^{-1}=a_{1}+\sum_{t=2}^{\min \{k, m\}} a_{t}\left(s_{k-t}^{(k)} s_{n}^{-1}\right)
$$

with $s_{j}^{(k)}:=s_{j}$ for $j>0$ and $s_{0}^{(k)}:=k$.

For $t<k$, we extend each quotient $s_{k-t}^{(k)} s_{n}^{-1}$ by every $s_{j}$ for which $k-t<j<n$ and $s_{j} \neq 0$ hold. Forming the quotients of every two of these $s_{j}$ succeeding each other, $s_{k-t}$ and $s_{n}$ included, we get the product of $q_{k-t}$ with each $q_{s}$ for which $k-t<s<n$ and $q_{s} \neq 0$. Writing these $q_{s}$ in the form $q_{s}^{(k)}$ and supplementing the missing $q_{s}$ with $k-t<s<n$ as factors $q_{s}^{(k)}$ with $q_{s}^{(k)}=1$, gives

$$
a_{t}\left(s_{k-t}^{(k)} s_{n}^{-1}\right)=a_{t} q_{k-t} \prod_{s=k-t+1}^{n-1} q_{s}^{(k)}=a_{t}^{(k)} \prod_{s=k-t}^{n-1} q_{s}^{(k)} .
$$

For $t=k$, the quotient $s_{0}^{(k)} s_{n}^{-1}$ can be rewritten analogously as a product with the factor $q_{0}^{(k)}=$ $k s_{k(0)}^{-1}=k\left(k(0) a_{k(0)}\right)^{-1}$. Then the modified sum is

$$
s_{n+1} s_{n}^{-1}=a_{1}+\sum_{t=2}^{\min \{n+1, m\}}\left(a_{t}^{(n+1)} \prod_{s=n+1-t}^{n-1} q_{s}^{(n+1)}\right)=p_{n, n+1} .
$$

Particularly, we have $p_{n, n+1} \neq 0$ if and only if $s_{n+1} \neq 0$. In this case we get $q_{n}=p_{n, n+1}^{-1}$.

Moreover, the equivalence of $s_{n+1}=0$ and $p_{n, n+1}=0$ constitutes the basis step of the proof by mathematical induction for the equivalence of the corresponding propositions $s_{j}=0$ and $p_{n, j}=0$ - each for $j=n+1, \ldots, k$ with $k<k(n)$. Here, $k(n) \leqslant n+m$ holds because otherwise (1) would lead to $s_{k(n)}=\sum_{j=1}^{m} a_{j} s_{k(n)-j}=0$ which contradicts the definition of $k(n)$.

For the inductive step, assume that the equivalence is already known for $j=n+1, \ldots, k-1$. From $s_{n} \neq 0$ and $s_{n+1}=\cdots=s_{k-1}=0$, it follows as above

$$
s_{k} s_{n}^{-1}=a_{k-n}+\sum_{t=k-n+1}^{\min \{k, m\}} a_{t}\left(s_{k-t}^{(k)} s_{n}^{-1}\right)=a_{k-n}+\sum_{t=k-n+1}^{\min \{k, m\}}\left(a_{t}^{(k)} \prod_{s=k-t}^{n-1} q_{s}^{(k)}\right)=p_{n, k} .
$$

Therefore the equivalence is also valid for $j=n+1, \ldots, k$. Particularly, we get $k(n)=\min \left\{k \in \mathbb{N}_{1} \mid\right.$ $k>n$ and $\left.p_{n, k} \neq 0\right\}$. Moreover, from $s_{n+1}=\cdots=s_{k(n)-1}=0$ it follows that $s_{k(n)} s_{n}^{-1}=p_{n, k(n)}$ which gives $q_{n}=p_{n, k(n)}^{-1}$.

The inequality $k(n-1)>n$ is equivalent with $s_{n}=0$ which leads to $k(n-1)=k(n)$ and $q_{n}=s_{n} s_{k(n)}^{-1}=0$.

\section{Error bounds and stopping criteria}

The error bounds and stopping criteria are based on the following result of Laguerre [8].

TheOREM 3. If $u \in \mathbb{C} \backslash\left(\mathcal{Z} \cup \mathcal{Z}_{1}\right)$, then every circle passing through $u$ and $h_{m}(u)$ has the property that either there are elements of $\mathcal{Z}$ inside and outside the circle or that all numbers of $\mathcal{Z}$ lie on the circle. 
Proof. We start from the well-known equation

$$
\frac{p^{\prime}(u)}{p(u)}=\sum_{j=1}^{k} \frac{m_{j}}{u-z_{j}} \quad \text { for } \quad u \in \mathbb{C} \backslash \mathcal{Z}
$$

With $v:=h_{m}(u)$, we get

$$
0=\sum_{j=1}^{k} m_{j}\left(\frac{a}{u-z_{j}}-\frac{a}{u-v}\right)
$$

for every $a \in \mathbb{C}$. If $\mathcal{C}$ is a circle through $u$ and $v$, then there exists a unique number $a \in \mathbb{C}$ with $|a|=1$, such that the injective function

$$
f:=\left(w \rightarrow \frac{a}{u-w}-\frac{a}{u-v}, w \in \mathbb{C} \backslash\{u\}\right)
$$

maps $v$ to $0, \mathcal{C} \backslash\{u\}$ to the real axis and the interior of $\mathcal{C}$ to the upper half-plane $\{z \in \mathbb{C} \mid \operatorname{Im} z>0\}$. Moreover, we have $0=\sum_{j=1}^{k} m_{j} f\left(z_{j}\right)$. Therefore either there is at least one element of $f(\mathcal{Z})$ in the upper half-plane and at least one in the lower half-plane or $f(\mathcal{Z})$ contains only real numbers. Then the mapping properties of $f$ yield the corresponding result with respect to the circle $\mathcal{C}$.

Especially, the closed disk

$$
\mathcal{L}_{u}=\mathcal{L}_{u}(p):=\left\{w \in \mathbb{C}|| w-h_{m / 2}(u)|\leqslant| u-h_{m / 2}(u) \mid\right\}
$$

which we call Laguerre disk of $u$, contains at least one root of $p$. The following theorem proves that the radius of $\mathcal{L}_{u}$ is less than $m\left|u-z_{j}\right|$, if $u$ is sufficiently close to $z_{j} \in \mathcal{Z}$. From this we obtain the stopping criterion in the first two stages.

THEOREM 4. Let $p(z)$ be a polynomial as specified above with $k \geqslant 2$. If $z_{j} \in \mathcal{Z}$, then

$$
\left|u-z_{j}\right|<m\left|\frac{p(u)}{p^{\prime}(u)}\right| \leqslant \frac{2 m-1}{2 m_{j}-1}\left|u-z_{j}\right|
$$

and $\mathcal{L}_{u} \cap \mathcal{Z}=\left\{z_{j}\right\}$ hold for every $u \in \mathbb{C}$ with $0<\left|u-z_{j}\right| \leqslant \mu_{j} /(2 m)$.

Proof. From (4) for $u \in \mathbb{C} \backslash \mathcal{Z}_{1}$, we get

$$
m\left|\frac{p(u)}{p^{\prime}(u)}\right|=\frac{m}{\left|m_{j}+S_{j}\right|}\left|u-z_{j}\right| \quad \text { with } \quad S_{j}:=\sum_{g \in \mathcal{J}_{k} \backslash\{j\}} m_{g} \frac{u-z_{j}}{u-z_{g}} .
$$

The assumption on $u$ leads to

$$
2 m\left|u-z_{j}\right| \leqslant\left|z_{g}-z_{j}\right| \leqslant\left|u-z_{g}\right|+\left|u-z_{j}\right| .
$$

This gives

$$
\frac{u-z_{j}}{u-z_{g}} \leqslant \frac{1}{2 m-1} \quad \text { for every } \quad g \in \mathcal{J}_{k} \backslash\{j\} .
$$

Then, with

$$
\left|S_{j}\right| \leqslant \frac{m-m_{j}}{2 m-1}
$$

we have

$$
m\left|\frac{p(u)}{p^{\prime}(u)}\right| \leqslant \frac{m}{m_{j}-\left|S_{j}\right|}\left|u-z_{j}\right| \leqslant \frac{2 m-1}{2 m_{j}-1}\left|u-z_{j}\right| .
$$


Now we assume that another zero $z_{l}$ with $l \in \mathcal{J}_{k} \backslash\{j\}$ is contained in the interior of $\mathcal{L}_{u}$. Then, with the abbreviation $d_{u}:=m\left|p(u) / p^{\prime}(u)\right|$ for the diameter of $\mathcal{L}_{u}$, we have $\left|z_{l}-h_{m / 2}(u)\right|<d_{u} / 2$ and $d_{u} \leqslant(1-1 /(2 m)) \mu_{j}$. It follows that

$$
\begin{aligned}
\left|z_{j}-z_{l}\right| & =\left|\left(z_{j}-u\right)+\left(\frac{1}{2} u-\frac{1}{2} h_{m}(u)\right)-\left(z_{l}-h_{m / 2}(u)\right)\right| \\
& \leqslant\left|z_{j}-u\right|+\frac{1}{2} d_{u}+\frac{1}{2} d_{u}<\frac{1}{2 m} \mu_{j}+\left(1-\frac{1}{2 m}\right) \mu_{j}=\mu_{j}
\end{aligned}
$$

which contradicts $\left|z_{j}-z_{l}\right| \geqslant \mu_{j}$. If there were no zeros in the interior of $\mathcal{L}_{u}$, then by Theorem 3 all zeros of $p$ must lie on the boundary of $\mathcal{L}_{u}$. This would give

$$
\left|z_{j}-z_{l}\right| \leqslant d_{u} \leqslant\left(1-\frac{1}{2 m}\right) \mu_{j}
$$

for every $l \in \mathcal{J}_{k} \backslash\{j\}$ contradicting $\left|z_{j}-z_{l}\right| \geqslant \mu_{j}$ as above. Hence $z_{j}$ is the only zero of $p$ in $\mathcal{L}_{u}$. Since $z_{j}$ lies in the interior of $\mathcal{L}_{u}$, the first inequality of (5) follows with Theorem 3 from

$$
\left|z_{j}-u\right|-\frac{1}{2} d_{u}=\left|z_{j}-u\right|-\left|u-h_{m / 2}(u)\right| \leqslant\left|z_{j}-h_{m / 2}(u)\right|<\frac{1}{2} d_{u}=\frac{m}{2}\left|\frac{p(u)}{p^{\prime}(u)}\right| .
$$

Since the a priori number of steps determined by (2) covers a wide range of "convergence quotients" $\left|z_{1} / z_{2}\right|$, it is desirable to have a simple pre-check instead of directly testing the stopping condition $m\left|p\left(q_{n}\right) / p^{\prime}\left(q_{n}\right)\right| \leqslant \varepsilon$ from (5). This can be arranged with the help of the following theorem.

THEOREM 5. Let $p(z)$ be a polynomial as specified above with $k \geqslant 3$. If $\left|z_{1}\right|<\left|z_{2}\right|<\left|z_{3}\right|$, then the sequences

$$
\left(\frac{q_{n+1}-q_{n+2}}{q_{n}-q_{n+1}}\right)_{n} \quad \text { and } \quad\left(\left(q_{n+1}-q_{n}\right) \frac{p^{\prime}\left(q_{n}\right)}{p\left(q_{n}\right)}\right)_{n}
$$

are convergent with limits $z_{1} / z_{2}$ and $m_{1}\left(z_{1} / z_{2}-1\right)$ respectively. From the second result it follows that

$$
\left|q_{n+1}-q_{n}\right|<2 m\left|\frac{p\left(q_{n}\right)}{p^{\prime}\left(q_{n}\right)}\right|
$$

holds for sufficiently large $n$.

Proof. Using the abbreviation $\tau_{g}:=m_{2}+\sum_{j=3}^{k} m_{j}\left(z_{2} / z_{j}\right)^{g}$ for $g \in \mathbb{N}_{1}$, we have

$$
q_{n}-z_{1}=z_{1} \zeta_{2}^{n} \frac{\tau_{n}-\zeta_{2} \tau_{n+1}}{m_{1}+\zeta_{2}^{n+1} \tau_{n+1}}
$$

from the proof of Theorem 1 and we get

$$
q_{n}-q_{n+1}=z_{1} \zeta_{2}^{n} \frac{m_{1}\left(\tau_{n}-2 \zeta_{2} \tau_{n+1}+\zeta_{2}^{2} \tau_{n+2}\right)+\zeta_{2}^{n+2}\left(\tau_{n} \tau_{n+2}-\tau_{n+1}^{2}\right)}{\left(m_{1}+\zeta_{2}^{n+1} \tau_{n+1}\right)\left(m_{1}+\zeta_{2}^{n+2} \tau_{n+2}\right)}
$$

Since $\lim _{n \rightarrow \infty} \tau_{n}=m_{2}$ and $\lim _{n \rightarrow \infty} \zeta_{2}^{n}=0$, it follows that $\left(\left(q_{n+1}-q_{n+2}\right) /\left(q_{n}-q_{n+1}\right)\right)_{n}$ is convergent with limit $\zeta_{2}=z_{1} / z_{2}$.

From (4), we obtain

$$
\left(q_{n+1}-q_{n}\right) \frac{p^{\prime}\left(q_{n}\right)}{p\left(q_{n}\right)}=m_{1} \frac{q_{n+1}-q_{n}}{q_{n}-z_{1}}+\left(q_{n+1}-q_{n}\right) \sum_{j=2}^{k} \frac{m_{j}}{q_{n}-z_{j}}
$$


As above, we find that $\left(\left(q_{n+1}-q_{n}\right) /\left(q_{n}-z_{1}\right)\right)_{n}$ converges to $\zeta_{2}-1$. Therefore we have

$$
\lim _{n \rightarrow \infty}\left(\left(q_{n+1}-q_{n}\right) \frac{p^{\prime}\left(q_{n}\right)}{p\left(q_{n}\right)}\right)=m_{1}\left(\frac{z_{1}}{z_{2}}-1\right) .
$$

With $m_{1} \leqslant m-2$ and $\left|z_{1} / z_{2}\right|<1$, it follows that there exists a number $n_{1}$ such that $\left|q_{n+1}-q_{n}\right|<$ $2 m\left|p\left(q_{n}\right) / p^{\prime}\left(q_{n}\right)\right|$ for every $n \in \mathbb{N}_{1}$ with $n \geqslant n_{1}$.

\section{The minimum method}

Let $n_{\varepsilon}$ be the bound on the right-hand side of (2). Even if the first $n_{\varepsilon}$ terms of the sequence $\left(q_{n}\right)_{n}$ don't approximate $z_{1}$ sufficiently, they can be used to approximate $\left|z_{1}\right|$ in the following way.

THEOREM 6. Let $z_{1}, \ldots, z_{k}$ be complex numbers as specified above, and let $h(n)$ be recursively defined by $h(0):=k(0)$ and $h(j+1):=k(h(j))$ for every $j \in \mathbb{N}_{0}$. If $c_{n}:=\left|m s_{h(n)}^{-1}\right|^{1 / h(n)}$ for $n \in \mathbb{N}_{0}$, then $\left|z_{1}\right|$ is the limit of the sequence $\left(\sigma_{n}\right)_{n}$ with

$$
\sigma_{n}:=\min \left\{s \in \mathbb{R} \mid \text { there exists a } j \in \mathbb{N}_{0} \text { with } h(j) \leqslant \max \{h(0), n\} \text { and } s=c_{j}\right\}
$$

for $n \in \mathbb{N}_{0}$ which consists of the "successive minima" of $\left(c_{n}\right)_{n}$. Moreover, $\left|z_{1}\right|$ is bounded by

$$
5^{-2^{-\nu}} \sigma_{m 2^{\nu}}<\left|z_{1}\right| \leqslant \sigma_{m 2^{\nu}} \quad \text { for each } \quad \nu \in \mathbb{N}_{0}
$$

The powers $c_{n}^{h(n)}$ can be calculated recursively by

$$
c_{0}^{h(0)}=\left|q_{0}\right|, \quad c_{n+1}^{h(n+1)}=c_{n}^{h(n)}\left|q_{h(n)}\right| \quad \text { for every } \quad n \in \mathbb{N}_{0} .
$$

Proof. By definition and using the triangle inequality, we get

$$
\left|s_{h(n)}\right|^{1 / h(n)}=\left|z_{1}\right|^{-1}\left|1+\left(\frac{z_{1}}{z_{2}}\right)^{h(n)}+\cdots+\left(\frac{z_{1}}{z_{m}}\right)^{h(n)}\right|^{1 / h(n)} \leqslant\left|z_{1}\right|^{-1} m^{1 / h(n)} .
$$

This gives

$$
\left|z_{1}\right| \leqslant c_{n} \quad \text { for each } n \in \mathbb{N}_{0} .
$$

Thus the monotone decreasing sequence $\left(\sigma_{n}\right)_{n}$ has the lower bound $\left|z_{1}\right|$. Therefore its convergence follows from the Bolzano-Weierstrass Theorem (on bounded monotone sequences). Hence to prove that $\left|z_{1}\right|$ is the limit of $\left(\sigma_{n}\right)_{n}$, it suffices to determine a subsequence having $\left|z_{1}\right|$ as limit. This can be done with the following result of Turán [10]. Let

$$
\sigma_{m 2^{\nu}}^{\star}:=\left(\max _{l \in \mathcal{J}_{m}}\left|\frac{1}{m} s_{l 2^{\nu}}\right|^{2^{-\nu} / l}\right)^{-1} \quad \text { for } \quad \nu \in \mathbb{N}_{0} .
$$

Then $\left|z_{1}\right|$ is bounded by

$$
5^{-2^{-\nu}} \sigma_{m 2^{\nu}}^{\star}<\left|z_{1}\right| \leqslant \sigma_{m 2^{\nu}}^{\star} \quad \text { for every } \quad \nu \in \mathbb{N}_{0} .
$$

The lower estimate with $\nu=0$ was found by Buchholtz. The general case was derived by Turán from Buchholtz's result. The proofs are given in [10].

If $\mathcal{N}_{\nu}:=\left\{l \in \mathcal{J}_{m} \mid s_{l 2^{\nu}} \neq 0\right\}$, then $\sigma_{m 2^{\nu}}^{\star}=\min _{l \in \mathcal{N}_{\nu}}\left|m s_{l 2^{\nu}}^{-1}\right|^{2^{-\nu} / l}$, and for each $l \in \mathcal{N}_{\nu}$ there exists a $j \in \mathbb{N}_{0}$ with $h(j) \leqslant m 2^{\nu}$ and $c_{j}=\left|m s_{l 2^{\nu}}^{-1}\right|^{2^{-\nu} / l}$. Therefore $\sigma_{m 2^{\nu}} \leqslant \sigma_{m 2^{\nu}}^{\star}$ for every $\nu \in \mathbb{N}_{0}$. Thus from (11) and (9), we get (8). Since $\lim _{\nu \rightarrow \infty} 5^{-2^{-\nu}}=1$, it follows that $\left|z_{1}\right|$ is the limit of the subsequence $\left(\sigma_{m 2^{\nu}}\right)_{\nu}$ and also of $\left(\sigma_{n}\right)_{n}$ as argued above. 
Since $(h(n))_{n}$ consists of the indices for which $s_{h(n)} \neq 0$ and since $s_{h(n+1)}=s_{k(h(n))}=s_{h(n)} /$ $q_{h(n)}$ for every $n \in \mathbb{N}_{0}$, the recursion formula for the powers $c_{n}^{h(n)}$ follows directly by substitution:

$$
c_{n+1}^{h(n+1)}=\left|m s_{h(n+1)}^{-1}\right|=\left|m s_{h(n)}^{-1} q_{h(n)}\right|=c_{n}^{h(n)}\left|q_{h(n)}\right| \quad \text { for every } \quad n \in \mathbb{N}_{0} .
$$

To find the indices of the successive minima, the powers in question are compared after crosswise exponentiation.

Every root of $p(z)$ generates a spherical point of the "analytical landscape" $\left\{(x, y, w) \in \mathbb{R}^{3} \mid w=\right.$ $\left.|p(x+i y)|^{2}\right\}$ which has no other hollows. Therefore we use the following method to obtain origin shifts of the current polynomial to good approximations of roots close to the "minimal distance circle" with radius $r:=\sigma_{n_{\varepsilon}}$ given by Theorem 6 .

If we look at the intersection of the analytical landscape and the cylinder over the circle with radius $r$ and with the origin as midpoint, we see that every local minimum of the function

$$
\pi_{r}:=\left(t \rightarrow|p(r \cos t+i r \sin t)|^{2}, t \in[0,2 \pi[)\right.
$$

belongs to a root in a hollow which produces the minimum. Therefore we proceed by approximating these minimum points. First we determine the coefficients

$$
f_{k}:=r^{k} \sum_{j=0}^{m-k} \operatorname{Re}\left(\bar{a}_{j+k} a_{j}\right) r^{2 j}, \quad g_{k}:=r^{k} \sum_{j=0}^{m-k} \operatorname{Im}\left(\bar{a}_{j+k} a_{j}\right) r^{2 j}, \quad k=1, \ldots, m,
$$

of the Fourier expansion

$$
\pi_{r}(t)-f_{0}=\sum_{k=1}^{m}\left(f_{k} 2 \cos (k t)+g_{k} 2 \cos \left(\frac{3 \pi}{2}+k t\right)\right) .
$$

Then we calculate the values of

$$
\pi_{r}^{\prime}(t)=\sum_{k=1}^{m}\left(k g_{k} 2 \cos (k t)-k f_{k} 2 \cos \left(\frac{3 \pi}{2}+k t\right)\right)
$$

at equidistant arguments $t$ using a table of $2 \cos (\pi n /(3 N))$ with $n=0, \ldots, 6 N-1$, which can be generated recursively by

$$
2 \cos ((n+2) \Delta)=(2 \cos \Delta)(2 \cos ((n+1) \Delta))-2 \cos (n \Delta)
$$

for $n=0, \ldots, 3 N-3$ with $\Delta:=\pi /(3 N)$, or for the first half of these values if $N$ is divisible by 4 . To enable easy doubling of this table using the same recursion and starting with $2 \cos (\Delta / 2)=$ $\sqrt{2 \cos \Delta+2}$ if necessary, we take powers of 2 for $N$ weakly depending on the degree of $p$. The factor 3 is introduced for the points $\xi_{i}$ in Section 5 .

Comparing every two successive values of $\pi_{r}^{\prime}(n \Delta)$ for $n=0, \ldots, 6 N-1$, we determine the arguments $x_{1}, \ldots, x_{e}$ with $\pi_{r}^{\prime}\left(x_{j}\right) \leqslant 0$ and $\pi_{r}^{\prime}\left(x_{j}+\Delta\right)>0, j \in\{1, \ldots, e\}$. These arguments generate approximations $r \cos x_{j}+i r \cos \left(3 \pi / 2+x_{j}\right), j=1, \ldots, e$, for the points belonging to the local minima of $\pi_{r}(t)$. Therefore the approximations are called minimum points. Then we choose these points as "origin shifts" for the current polynomial and calculate the diameter of the Laguerre disks for the "stopping values" of the corresponding Bernoulli sequences. Each of the values for which the Laguerre diameter does not exceed the desired accuracy $\varepsilon$ is used as an approximation of a zero. If all diameters are greater than $\varepsilon$, we continue in Section 5 with the value which leads to the minimal diameter.

Each minimum distance circle together with all minimum points is called minimum circle. Since at least those roots which have caused the bad behavior of the Bernoulli sequence in the first phase are close to the minimum circle, we diminish the stepfactor $\left(\log \varkappa^{-1}\right)^{-1}$ on the right-hand side of (2) to 2.0, as announced on p. 54 . 


\section{Chained minimum circles and modified Turán circles}

In Theorem 6 , we proved that $\left|z_{1}\right|$ is the limit of a sequence which can be calculated with the help of a Bernoulli sequence $\left(q_{n}\right)_{n}$. Since we stop at a relatively small number of terms approximating the radius of the minimum circle, we can't be sure to find a root this way, despite the good numerical experience. To fill this gap in Stage One of the SPA, we proceed in the following two ways.

On the minimum circle with radius $r$ let $\zeta$ be a minimum point with minimal Laguerre diameter, as introduced in Section 4. Then we shift the actual polynomial with $\zeta$ and calculate the radius $r_{1}$ of the minimum circle for the shifted polynomial. If $r_{1}<0.3 r$, we proceed with the new circle as in Section 4. If these steps are repeated until an approximation of a zero is obtained, we get chained minimum circles.

The finiteness of such a chain can be proved as follows. After $n$ repetitions with $r_{i}<0.3 r_{i-1}$, $i=1, \ldots, n, r_{0}:=r$, we have $r_{n}<0.3^{n} r$. Since all minimum circles contain a zero, and since $\left(0.3^{n} r\right)_{n}$ converges to 0 , there would exist an exponent $e$, such that all points on the $e$-th circle of the chain fulfill the condition of Theorem 4 . Therefore the chain consists of at most $e+1$ circles.

If there would be two successive minimum circles with minimal Laguerre diameters and with radii $r$ and $r_{1}$ fulfilling $r_{1} \geqslant 0.3 r$, which, up to now, never occurred, then the ultimate case is based on the following idea of Turán [10] who developed a whole method of rootfinding with it. At first, the number of steps for the Bernoulli iterations is chosen as $16 \mathrm{~m}$, until the next root is approximated. With respect to (8) instead of (11), we call a set

$$
\mathcal{T}(\xi):=\left\{z \in \mathbb{C}|| z-\xi \mid=\sigma_{16 m}\right\}
$$

modified Turán circle, if in the case $\xi \neq 0$, the actual polynomial is shifted with $\xi$ before calculating $\sigma_{16 m}$.

The minimum circle with radius $r$, which we considered above, is replaced by $\mathcal{T}(0)$ with radius $r^{\prime}:=\sigma_{16 m}$, containing a zero $z_{1}$ with

$$
0.9 r^{\prime}<\sqrt[16]{0.2} r^{\prime}<\left|z_{1}\right|<r^{\prime}
$$

by (8). The annulus $\left\{z \in \mathbb{C}\left|0.9 r^{\prime}<\right| z \mid<r^{\prime}\right\}$ is covered by 12 disks $\left\{z \in \mathbb{C}|| z-\xi_{j} \mid \leqslant \vartheta r^{\prime}\right\}$ with $\xi_{j}:=\left(19 r^{\prime} / 20\right)(\cos (j \pi / 6)+i \sin (j \pi / 6)), j=0, \ldots, 11$, and $\vartheta:=\sqrt{1 / 400+(19 / 5) \sin ^{2}(\pi / 24)}$. Hence $z_{1}$ is contained in one of these disks. If $\xi_{k}$ is the midpoint of this disk, then $\mathcal{T}\left(\xi_{k}\right)$ has a radius $r_{1}^{\prime}$ with $0.9 r_{1}^{\prime}<\left|z_{1}\right|<\vartheta r^{\prime}$. Therefore we get $r_{1}^{\prime}<10 \vartheta r^{\prime} / 9<0.3 r^{\prime}$ [10, pp. 302-305].

Now the SPA is continued as in Section 4 with $\mathcal{T}\left(\xi_{k}\right)$ as a minimum circle. The resulting chain may contain further modified Turán circles. But each circle has a radius smaller than 0.3 times the preceding radius. The same conclusions as above prove that these chains are finite and therefore that the SPA is reliable. At the same time, the following conjecture of Euler $[11, \S 340]$ is confirmed: For each root, a substitution of the given polynomial can be determined substantially using Bernoulli sequences, such that, with the new polynomial, a Bernoulli sequence approximates the root.

\section{Separation of the roots}

At the end of Stage One we obtain a list containing as triples the approximations of all roots, the diameters of the corresponding Laguerre disks calculated for the original polynomial and the minimal distances of these disks fom the other ones. The following example, received by [9], is commented in detail in [12].

The result at the end of Stage One for the polynomial

$$
\begin{gathered}
\left(\left(z-i+10^{-11}\right)^{3}-10^{-10}\right) \cdot\left(\left(z-i-10^{-13}\right)^{4}+2 \cdot 10^{-13}\right) \\
-\left(\left(z-i+2 \cdot 10^{-15}\right)^{4}\right) \cdot\left(\left(z-i+10^{-14}\right)^{2}+10^{-10}\right)
\end{gathered}
$$


is

$$
\begin{gathered}
{[[0.00077353032739554464549+1.0006770160484033276 i,} \\
0.00019165867790659214168,0.00065554810623314565385], \\
{[-0.00019885329521704972714+1.0010084693218859346 i,} \\
\left.0.5969694786440298130110^{-5}, 0.00082969396329870887029\right], \\
{[0.00097380641505493865706+0.99966860219052214291 i,} \\
0.00018090265558687391027,0.00065554810623314565385], \\
{[-0.00097303914697718310885+1.0003326680812441389 i,} \\
0.000016647250865373317460,0.00079995617086085220203], \\
{[-0.00077494745909348685614+0.99932292694377297523 i,} \\
0.00021238515037269297347,0.00075618008195395334603], \\
{[1.0000000001702279985+1.0000000000000000037 i,} \\
\left.0.6999999990054428266010^{-18}, 0.99884534606536475640\right], \\
{[0.00019950295900923792091+0.99899031741417147720 i,} \\
0.000061086512935947270899,0.00075618008195395334603]] .
\end{gathered}
$$

If every two disks are disjoint and if the diameters are less then the desired accuracy, we are ready. Sometimes, this is not true, since by default we start with medium accuracy to get an overview. Due to the deflation error it may happen that we miss roots located outside the Laguerre disks while others are associated with more than one disk.

Therefore, the main goal of Stage Two is to ameliorate the approximations so that all roots will be contained in Laguerre disks with sufficiently small diameters. Principally, these results are obtained by stepwise increasing the precision of the basic operations and the accuracy of the approximations which can be achieved dynamically with most computer algebra systems.

Since every Laguerre disk contains at least one root, the ameliorations are always carried out with the SPA after an origin shift of the given polynomial to the center of the Laguerre disk. Moreover, to avoid repeated calculation of the same root we look out for all other Laguerre disks which contain this root. Such a root is stored with the disk data and will be deflated before the SPA is used to search for further roots in that disk.

This process is repeated until all zeros in each Laguerre disk are determined. Since the sum of the multiplicities of the roots contained in a disk can be calculated with the help of the Cohn-Schur transformation (cf. [6]), we have a precise stopping condition.

If having passed all Laguerre disks we miss some roots due to the deflation error in Stage One, we go on with deflating the given polynomial with the known roots one after the other. Then the resulting polynomial is treated as in Stage One. The following theorem shows that by increasing the accuracy, the deflation error can be made as small as it is necessary to locate all roots of the given polynomial in disjoint Laguerre disks.

THEOREM 7. Let $f(z)$ be a polynomial, $w$ be a root of $f(z)$ with multiplicity $g, \eta$ be a nonzero complex number and

$$
\delta=\delta(w, \eta):=\min \{t \in \mathbb{R} \mid \text { there is a } v \in \mathbb{C} \text { with } f(v)=\eta \text { and }|v-w|=t\}
$$

If $|\eta|$ is sufficiently small, then

$$
\delta \leqslant\left(\frac{2 g !|\eta|}{\left|f^{(g)}(w)\right|}\right)^{1 / g} .
$$


Proof. Let $u \in \mathbb{C}$ be a solution of $f(u)=\eta$ with $|u-w|=\delta$. By elementary methods it can be shown that

$$
f(u)-f(w)-\frac{1}{1 !} f^{\prime}(w)(u-w)-\cdots-\frac{1}{g !} f^{(g)}(w)(u-w)^{g}=q(u, w)(u-w)^{g+1}
$$

with an explicitly known polynomial $q(u, w)$. Here we have

$$
f(u)=\eta, \quad f(w)=f^{\prime}(w)=\cdots=f^{(g-1)}(w)=0, \quad f^{(g)}(w) \neq 0 .
$$

If $f(z)$ has degree $m$ and if $b_{m}$ is the coefficient of $z^{m}$, then Vieta's root theorem leads to

$$
\delta=|u-w| \leqslant\left|\eta b_{m}^{-1}\right|^{1 / m} .
$$

This can be ameliorated in the following way. There are well known upper bounds $B$ of $|w|$ depending on the coefficients of $f(z)$. Restricting $|\eta|$ by an upper bound $M>0$, we may take $B+\left(M\left|b_{m}^{-1}\right|\right)^{1 / m}$ as an upper bound for $|u|$ by (13). Therefore, for every $M>0$, a uniform upper bound $K>0$ of $|q(u, w)|$ exists such that

$$
\left|\eta-\frac{1}{g !} f^{(g)}(w)(u-w)^{g}\right| \leqslant K \delta^{g+1}
$$

for every $\eta$ with $|\eta| \leqslant M$. It follows that

$$
\delta^{g}\left(\frac{1}{g !}\left|f^{(g)}(w)\right|-K \delta\right) \leqslant|\eta| .
$$

If

$$
|\eta| \leqslant \min \left\{\frac{\left|f^{(g)}(w)\right|^{m}}{(2 g ! K)^{m}}\left|b_{m}\right|, M\right\},
$$

then (13) gives $\delta \leqslant\left|f^{(g)}(w)\right| /(2 g ! K)$. Now, from (14) we obtain

$$
\delta^{g} \frac{\left|f^{(g)}(w)\right|}{2 g !} \leqslant|\eta|
$$

which is equivalent with (12).

The SPA uses $\eta$ in the following way. If $w_{0}$ is another zero of $f(z)$ and if $w_{0}^{*}$ is an approximation of $w_{0}$ determined with the stopping condition

$$
m\left|\frac{f\left(w_{0}^{*}\right)}{f^{\prime}\left(w_{0}^{*}\right)}\right| \leqslant \varepsilon,
$$

where $\varepsilon$ is the desired upper bound of $\left|w_{0}-w_{0}^{*}\right|$, then deflation with $w_{0}^{*}$ leads to a polynomial $f_{1}^{*}(z)$ satisfying

$$
f(z)=\left(z-w_{0}^{*}\right) f_{1}^{*}(z)+f\left(w_{0}^{*}\right) .
$$

With $\eta:=f\left(w_{0}^{*}\right)$ Theorem 7 gives an upper bound for the deflation error $|u-w|=\delta$, where $u$ is the zero of $f(z)-\eta=\left(z-w_{0}^{*}\right) f_{1}^{*}(z)$ corresponding to $w$.

By (15) we have $|\eta| \leqslant\left|f^{\prime}\left(w_{0}^{*}\right)\right|(\varepsilon / m)$. Therefore, by repeated application of Theorem 7 , the total deflation error $\delta_{d}(\varepsilon)$ after $d$ deflations fulfills

$$
\left|\delta_{d}(\varepsilon)\right| \leqslant \sum_{i=1}^{d} c_{i} \varepsilon^{1 / g_{i}}
$$


with nonnegative constants $c_{i}$ only depending on $f$ and with multiplicities $g_{i}$ which are defined successively by Theorem 7 for the sequence of roots in the order of the corresponding deflations.

Now, let $w$ be any zero of $p, w^{*}$ be the corresponding zero of a deflation polynomial after $d$ deflations and $u$ be an approximation of $w^{*}$. By stepwise decreasing $\varepsilon$, the inequality

$$
\max \left\{\varepsilon,\left|\delta_{d}(\varepsilon)\right|\right\} \leqslant \frac{1}{4 m} \mu
$$

can be satisfied which leads to $\left|u-w^{*}\right| \leqslant \mu /(4 m)$ by (15) and to $\left|w-w^{*}\right| \leqslant \mu /(4 m)$ by (16). Therefore we get $|u-w| \leqslant \mu /(2 m)$ and Theorem 4 gives $\mathcal{L}_{u} \cap \mathcal{Z}=\{w\}$.

As mentioned above this enables us to determine disjoint Laguerre disks which contain all roots. If $w$ has multiplicity $g>1$, then there may be $g$ different approximations of $w$ which generate up to $g$ Laguerre disks. All these disks contain $w$ but no other roots. Moreover, $w$ is an interior point of the intersection. Therefore, the approximations of $w$ can be ameliorated locally so that they finally lie in only one of the disks.

The practical use of condition (17) raises two problems. The deflation error $\delta_{d}(\varepsilon)$ is not known explicitly and the minimal distance $\mu$ of the roots can only be approximated in a complicated manner. In [1] a simple lower bound $\mu^{\prime}$ of $\mu$ is given. But, up to now, this result can't be used efficiently because the method is either very time-consuming or extremely sensitive to roundoff errors. Moreover, the approximation of all roots in Stages One and Two often runs more than twice as fast as the calculation of the rational number $\left(\mu^{\prime}\right)^{2}$ even using integer arithmetic. Therefore, we never estimate the minimal distance $\mu$ of the roots in advance. Instead of that, the precision of the operations and the accuracy of the results are ameliorated stepwise with $\varepsilon=10^{-[2+\log m] j}$, $j=1, \ldots, J$, where the stopping number $J$ is defined through successful separation of the roots which is guaranteed by the above considerations.

From (16) and (17) with

$$
\gamma:=\max \left\{g \in \mathbb{N}_{1} \mid \text { there is an } i \in \mathcal{J}_{d} \text { with } g=g_{i}\right\}
$$

we get $J=O(\gamma \log (1 / \mu))$, where $\gamma$ does not really depend on $m$ because the multiplicities $g_{i}$ are determined by successive deflation polynomials. Since we use quotients of power sums, shifts and deflations as in Stage One, the worst case complexity in Stage Two is $O\left(m^{4} \log (1 / \mu)\right)$. Moreover, it should be mentioned that two shifts are as costly as $m$ deflations with decreasing degree.

\section{Newton-Raphson iteration}

This optional Stage Three provides a simple iterative method to ameliorate the approximations. In the case of roots with multiplicity 1 the convergence is at least quadratic. If a Laguerre disk $\mathcal{L}$ contains only one root $z_{j}$ with any multiplicity $m_{j}$ and if $\mathcal{L}$ has no common elements with the other Laguerre disks determined in Stage Two, then the minimal distance between $\mathcal{L}$ and the other Laguerre disks is a lower bound for $\mu_{j}$ in the following theorem.

THEOREM 8. Let $p(z)$ be a polynomial as specified above with $k \geqslant 2$. If $z_{j} \in \mathcal{Z}$, then

$$
\left|h_{m_{j}}(u)-z_{j}\right| \leqslant \frac{m-m_{j}}{2 m m_{j}-m}\left|u-z_{j}\right|
$$

holds for every $u \in \mathbb{C}$ with $0<\left|u-z_{j}\right| \leqslant \mu_{j} /(2 m)$.

The sequence $\left(u_{n}\right)_{n}$ with $u_{0}:=u$ and $u_{n+1}:=h_{m_{j}}\left(u_{n}\right)$ for $n \in \mathbb{N}_{0}$ converges to $z_{j}$. The error can be estimated a posteriori by

$$
\left|u_{n+1}-z_{j}\right| \leqslant \frac{m-m_{j}}{2 m\left(m_{j}-1\right)+m_{j}}\left|u_{n}-u_{n+1}\right| \quad \text { for every } n \in \mathbb{N}_{0} .
$$


If $m_{j}=1$, the convergence is at least quadratic which means that there exists a constant $K_{1}>0$ such that

$$
\left|u_{n+1}-z_{j}\right| \leqslant K_{1}\left|u_{n}-z_{j}\right|^{2} \quad \text { for every } n \in \mathbb{N}_{0} .
$$

Proof. Similar to the proof of Theorem 4 we get

$$
\left|h_{m_{j}}(u)-z_{j}\right|=\left|1-\frac{m_{j}}{m_{j}+S_{j}}\right|\left|u-z_{j}\right| \leqslant \frac{\left|S_{j}\right|}{m_{j}-\left|S_{j}\right|}\left|u-z_{j}\right| \leqslant \frac{m-m_{j}}{2 m m_{j}-m}\left|u-z_{j}\right|=: q\left|u-z_{j}\right| .
$$

With

$$
q=\frac{1}{2 m_{j}-1}\left(1-\frac{m_{j}}{m}\right)<1
$$

mathematical induction leads to

$$
\left|u_{n}-z_{j}\right| \leqslant\left|u_{0}-z_{j}\right| q^{n} \leqslant \frac{1}{2 m} \mu_{j} q^{n} \quad \text { for all } \quad n \in \mathbb{N}_{0}
$$

which means that $z_{j}$ is the limit of $\left(u_{n}\right)_{n}$.

From (18), we have

$$
\left|u_{n+1}-z_{j}\right| \leqslant q\left|u_{n}-z_{j}\right| \leqslant q\left|u_{n}-u_{n+1}\right|+q\left|u_{n+1}-z_{j}\right|
$$

and therefore

$$
\left|u_{n+1}-z_{j}\right| \leqslant \frac{q}{1-q}\left|u_{n}-u_{n+1}\right|
$$

which is equivalent with (19).

Since $p^{\prime}(u) \neq 0$ for every $u \in \mathbb{C}$ with $\left|u-z_{j}\right| \leqslant \mu_{j} /(2 m)$, the function $h_{1}(u)$ is infinitely often differentiable at $z_{j}$. Therefore a constant $K_{1}>0$ exists such that

$$
\left|h_{1}(u)-h_{1}\left(z_{j}\right)-h_{1}^{\prime}\left(z_{j}\right)\left(u-z_{j}\right)\right| \leqslant K_{1}\left(u-z_{j}\right)^{2} .
$$

With $h_{1}\left(z_{j}\right)=z_{j}$ and $h_{1}^{\prime}\left(z_{j}\right)=0$ it follows that

$$
\left|h_{1}(u)-z_{j}\right| \leqslant K_{1}\left|u-z_{j}\right|^{2} .
$$

\section{Conclusion}

The following results concerning Bernoulli sequences, Laguerre disks, minimum circles, and modified Turán circles are obtained in this paper.

1. A lower bound for the number of steps of a Bernoulli sequence to approximate a zero with smallest modulus is derived for prescribed accuracy.

2. A recursion formula for the Bernoulli sequences and properties of the Laguerre disks needed for stopping criteria and error estimates are proved as in [1].

3. Chained minimum circles are combined with modified Turán circles to guarantee the reliability of the SPA. Since the radii of all circles are calculated with the help of Bernoulli sequences, at the same time a conjecture of Euler [11] is confirmed, concerning the determination of substitutions for the given polynomial to approximate all zeros, substantially using Bernoulli sequences.

\section{Bibliography}

[1] H. Möller, Algorithmische Lineare Algebra, Verlag Vieweg, Braunschweig, 1997; http:// www math.uni-muenster.de/u/mollerh. 
[2] M. A. Jenkins, J.F. Traub, "Principles for testing polynomial zerofinding programs", ACM Trans. Math. Software, 1:1 (1975), 26-34.

[3] D. V. Chudnovsky, G. V. Chudnovsky, "Computer algebra in the service of mathematical physics and number theory", Computers in Mathematics, Lecture Notes in Pure and Appl. Math., 125, Marcel Dekker, New York, 1990, 109-232.

[4] M. A. Jenkins, J. F. Traub, "A three-stage variable-shift iteration for polynomial zeros and its relation to generalized Rayleigh iteration", Numer. Math., 14:3 (1970), 252-263.

[5] T. E. Hull, R. Mathon, "The mathematical basis and a prototype implementation of a new polynomial rootfinder with quadratic convergence", ACM Trans. Math. Software, 22:3 (1996), 261-280.

[6] D. Loewenthal, "Improvements on the Lehmer-Schur root detection method", J. Comput. Phys., 109:2 (1993), 164-168.

[7] A. Schönhage, "Equation solving in terms of computational complexity", Proceedings of the International Congress of Mathematicians, Amer. Math. Soc., Providence, RI, 1987, 131-153.

[8] E. Laguerre, Euvres, Tome I, Gauthier-Villars, Paris, 1898.

[9] H. Möller, SPAview.mws, Maple worksheet; http://www.math.uni-muenster.de/u/mollerh.

[10] P. Turán, On a New Method of Analysis and Its Applications, John Wiley \& Sons, New York, 1978.

[11] L. Euler, Introductio in Analysin Infinitorum, M.-M. Bousquet, Lausanne, 1748; Introduction to Analysis of the Infinite, Book I, Springer-Verlag, Berlin, 1988.

[12] H. Möller, Visualization of the First Stage of the SPA, Report of [9]; http://www.math.unimuenster.de/u/mollerh. 